\title{
REDUCTION OF SYSTEMS OF LINEAR EQUATIONS IN ORDINAL VARIABLES
}

\author{
J. L. HICKMAN ${ }^{1}$
}

ABSTRACT. In this note we are concerned with a general finite system

$$
\sum_{i=0}^{n-1} x_{i} \alpha_{j i}=\beta_{j} ; \quad j<m,
$$

of $m$ linear equations in $n$ variables, where the $\alpha_{j i}$ and the $\beta_{j}$ are positive ordinals, and the variables $x_{i}$ range over ordinals.

In the particular case $n=1$ we show that (S) can be reduced to a canonical form $\left(\mathrm{S}^{*}\right)$ having solutions of a relatively simple type, and we use $\left(\mathrm{S}^{*}\right)$ to obtain the solution-set of $(\mathrm{S})$.

In the general case we show that $(S)$ can be reduced to a finite sequence of single-variable systems, and again obtain the solution-set of (S) in terms of the solution-sets of these simpler systems.

We assume a knowledge of the elementary theory of ordinal arithmetic, such as may be found for example in [2].

Ordinals will generally be denoted by lower-case Greek letters, with finite ordinals (natural numbers) being denoted by lower-case Latin letters; the first transfinite ordinal will always be denoted by " $\omega$ ".

For $\alpha>0$, let $\alpha=\sum_{i=0}^{n} \omega^{e_{i}(\alpha)} c_{i}(\alpha)$ be the (Cantor) normal form of $\alpha$. We put $l(\alpha)=n+1$ (the "length" of $\alpha$ ), $e(\alpha)=e_{0}(\alpha)$ (the "degree" of $\alpha$ ), $c(\alpha)=c_{n}(\alpha)$, and $r(\alpha)=\omega^{e_{n}(\alpha)}$. Of course $r(\alpha)$ is the smallest positive remainder of $\alpha$, and $\alpha$ is a successor if and only if $r(\alpha)=1$. Finally, if $r(\alpha)=1$, then we put $I(\alpha)=\sum_{i=0}^{n-1} \omega^{e_{i}(\alpha)} c_{i}(\alpha)$.

We note that for all $\alpha, \beta>0$ we have either $l(\alpha \beta)=l(\beta)$ or else $l(\alpha \beta)=l(\alpha)+l(\beta)-1$, and that $l(\alpha \beta)=l(\beta)$ if and only if either $l(\alpha)$ $=1$ or $r(\beta)>1$. These facts can easily be verified by expanding $\alpha$ and $\beta$ into normal form and multiplying out.

We require the following results on right-divisors of ordinals; these are (in essence) set forth in [1].

Result 1. A nonzero limit ordinal $\alpha$ is a right-divisor of a nonzero (limit) ordinal $\beta$ if and only if the following hold:

(a) $l(\alpha)=l(\beta)$

(b) There is some $\delta<e(r(\beta))$ such that $\delta+e_{i}(\alpha)=e_{i}(\beta)$ and $c_{i}(\alpha)$ $=c_{i}(\beta)$ for every $i<l(\alpha)$.

In connection with the above, we note that if $\alpha$ is a nonzero limit ordinal and $\delta$ is any ordinal, then $\psi \alpha=\omega^{\delta} \alpha$ for any ordinal $\psi$ with $\omega^{\delta} \leqslant \psi<\omega^{\delta+1}$.

Received by the editors November 19, 1974 and, in revised form, July 24, 1975.

AMS (MOS) subject classifications (1970). Primary 04A10.

1 The work contained in this paper was done whilst the author held a Research Fellowship at the Australian National University. 
For we have $\psi=\omega^{\delta} c+\gamma$ for some positive number $c$ and some ordinal $\gamma<\omega^{\delta}$, and we also have $\alpha=\omega \theta$ for some positive ordinal $\theta$. But then $\psi \alpha=\left(\left(\omega^{\delta} c+\gamma\right) \omega\right) \theta=\left(\left(\omega^{\delta} c\right) \omega\right) \theta=\omega^{\delta} \omega \theta=\omega^{\delta} \alpha$.

Result 2. Let $\alpha$ be a successor ordinal of length $p+2$. Then $\alpha$ is a rightdivisor of a nonzero ordinal $\beta$ if and only if the following hold:

(a) $l(\beta) \geqslant p+2$

(b) $c(\alpha)$ divides $c_{p+1}(\beta)$;

(c) $\omega^{e_{p+1}(\beta)} I(\alpha)=\sum_{i=0}^{p} \omega^{e_{i}(\beta)} c_{i}(\beta)$.

In this case the equation $x \alpha=\beta$ has a unique solution.

Consider the system

$$
x \alpha_{j}=\beta_{j} ; \quad j<m,
$$

of $m$ linear equations in 1 variable. Obviously if (S1) has a solution, then we have

$$
\alpha_{j} \text { right-divides } \beta_{j} ; \quad j<m .
$$

THEOREM 1. Let the system

$$
x \alpha_{j}=\beta_{j} ; \quad j<m,
$$

of $m$ linear equations in 1 variable satisfy condition $(\mathrm{C} 1)$. Then there is a system

$$
y \alpha_{j}=\tau_{j} ; \quad j<m
$$

such that:

(1) $l\left(\alpha_{j}\right)=l\left(\tau_{j}\right)$ for every $j<m$;

(2) If $\left(\mathrm{S}^{*}\right)$ has a solution, then it has a solution $y=\omega^{\delta}$ for some $\delta$;

(3) (S1) has a solution if and only if the following hold:

(I) $\left(\mathrm{Si}^{*}\right)$ has a solution;

(II) For all $i, j<m$ such that $r\left(\alpha_{i}\right)=r\left(\alpha_{j}\right)=1$ we have

$$
c\left(\alpha_{i}\right) c_{q_{j}}\left(\beta_{j}\right)=c\left(\alpha_{j}\right) c_{q_{i}}\left(\beta_{i}\right),
$$

where $q_{i}=l\left(\alpha_{i}\right)-1$ and $q_{j}=l\left(\alpha_{j}\right)-1$;

$$
\begin{aligned}
\sum\left\{\omega^{e_{k}\left(\beta_{i}\right)} c_{k}\left(\beta_{i}\right) ; l\left(\alpha_{i}\right)\right. & \left.\leqslant k<l\left(\beta_{i}\right)\right\} \\
= & \sum\left\{\omega^{e_{k}\left(\beta_{j}\right)} c_{k}\left(\beta_{j}\right) ; l\left(\alpha_{j}\right) \leqslant k<l\left(\beta_{j}\right)\right\} .
\end{aligned}
$$

Proof. We define the $\tau_{j}$ as follows. If $r\left(\alpha_{j}\right)>1$, put $\tau_{j}=\beta_{j}$. Otherwise, put

$$
\tau_{j}=\left(\sum_{i=0}^{p-1} \omega^{e_{i}\left(\beta_{j}\right)} c_{i}\left(\beta_{j}\right)\right)+\omega^{e_{p}\left(\beta_{j}\right)} c\left(\alpha_{j}\right)
$$

where $p=l\left(\alpha_{j}\right)-1$.

This defines $\left(\mathrm{Sl}^{*}\right)$ : since $(\mathrm{S} 1)$ satisfies $(\mathrm{C} 1)$, it follows from Result 1 that $\left(\mathrm{S} 1^{*}\right)$ satisfies condition (1).

We now consider two cases.

(A) $r\left(\alpha_{j}\right)>1$ for every $j<m$. From the above definition of the $\tau_{j}$ we see that $\left(\mathrm{Sl}^{*}\right)$ is simply $(\mathrm{S} 1)$, and from this and our assumption on the $r\left(\alpha_{j}\right)$, 
condition (3) follows trivially. It therefore suffices to demonstrate (2), and so we assume that $\left(\mathrm{S}^{*}\right)$ has a solution, $y=\psi$. Now there is an ordinal $\delta$ such that $\omega^{\delta} \leqslant \psi<\omega^{\delta+1}$; since $r\left(\alpha_{j}\right)>1$ for each $j$, the remark following Result 1 tells us that $\psi \alpha_{j}=\omega^{\delta} \alpha_{j}$ for each $j$. Thus $y=\omega^{\delta}$ is also a solution.

(B) $r\left(\alpha_{j}\right)=1$ for some $j<m$.

We commence by proving (2); thus let $y=\psi$ be a solution of $\left(\mathrm{S} 1^{*}\right)$. From the remark following Result 2 , we see that $y=\psi$ is the only solution of $\left(\mathrm{S}^{*}\right)$, and so we must show that $\psi=\omega^{\delta}$ for some $\delta$.

Choose $j^{\circ}<m$ such that $r\left(\alpha_{j^{\circ}}\right)=1$. From (1) we have $l\left(\alpha_{j^{\circ}}\right)=l\left(\tau_{j^{\circ}}\right)$ $=l\left(\psi \alpha_{j^{\circ}}\right)$, and so from the remark preceding Result 1 we have that $l(\psi)=1$, that is, $\psi=\omega^{\delta} b$ for some $\delta$ and some nonzero number $b$. However, we also have $b c\left(\alpha_{j^{\circ}}\right)=c\left(\psi \alpha_{j^{\circ}}\right)=c_{p}\left(\tau_{j^{\circ}}\right)=c\left(\alpha_{j^{\circ}}\right)$, where $p=l\left(\alpha_{j^{\circ}}\right)-1$. Hence $b$ $=1$, and so $\psi=\omega^{\delta}$ as desired.

It remains to demonstrate condition (3). Thus suppose that $x=\psi$ is a solution of (S1), and let $\delta$ be such that $\omega^{\delta} \leqslant \psi<\omega^{\delta+1}$. If $r\left(\alpha_{j}\right)>1$, then as before we have $\omega^{\delta} \alpha_{j}=\psi \alpha_{j}=\beta_{j}=\tau_{j}$. If, on the other hand, $r\left(\alpha_{j}\right)=1$, then we have $\omega^{\delta} \alpha_{j}=\omega^{\delta} I\left(\alpha_{j}\right)+\omega^{\delta} c\left(\alpha_{j}\right)$ : we must show that the right side of this equation is in fact $\tau_{j}$. Put $\psi=\omega^{\delta} b+\gamma$ for some nonzero number $b$ and some $\gamma<\omega^{\delta} ;$ then $\beta_{j}=\psi \alpha_{j}=\omega^{\delta} I\left(\alpha_{j}\right)+\omega^{\delta} b c\left(\alpha_{j}\right)+\gamma$. From this it is easily seen that if we set $p=l\left(\alpha_{j}\right)-1$, then

$$
\delta=e_{p}\left(\beta_{j}\right) \text { and } \omega^{\delta} I\left(\alpha_{j}\right)=\sum_{i=0}^{p-1} \omega^{e_{i}\left(\beta_{j}\right)} c_{i}\left(\beta_{j}\right) .
$$

Hence we do indeed have $\omega^{\delta} \alpha_{j}=\tau_{j}$. Thus $y=\omega^{\delta}$ is a solution of $\left(\mathrm{S} 1^{*}\right)$, and so (I) holds.

With $x=\psi$ as above, we now have to demonstrate (II), and so we take $i, j<m$ and assume that $r\left(\alpha_{i}\right)=r\left(\alpha_{j}\right)=1$. As above we have $\beta_{j}=\omega^{\delta} I\left(\alpha_{j}\right)$ $+\omega^{\delta} b c\left(\alpha_{j}\right)+\gamma$, with of course a similar expression for $\beta_{i}$. Thus $\beta_{i}, \beta_{j}$ have the common remainder $\gamma$, and since $l(\gamma)=l\left(\beta_{j}\right)-l\left(\alpha_{j}\right)=l\left(\beta_{i}\right)-l\left(\alpha_{i}\right)$, this establishes (IIb). But the same expressions for $\beta_{i}$ and $\beta_{j}$ tell us that $c_{q_{i}}\left(\beta_{i}\right) / c\left(\alpha_{i}\right)=b=c_{q_{j}}\left(\beta_{j}\right) / c\left(\alpha_{j}\right)$, with $q_{i}, q_{j}$ as in (IIa). This establishes (IIa).

Suppose now that (I) and (II) hold, and let $y=\sigma$ be a solution of $\left(\mathrm{Sl}^{*}\right)$. From our assumption that $r\left(\alpha_{j}\right)=1$ for some $j<m$ it follows that $y=\sigma$ is the only solution of $\left(\mathrm{Sl}^{*}\right)$, and so from (2), which has already been established, we conclude that $\sigma=\omega^{\delta}$ for some $\delta$.

Take a particular $i<m$ for which $r\left(\alpha_{i}\right)=1$, and put $p=l\left(\alpha_{i}\right)-1, q$ $=l\left(\beta_{i}\right)-1$. Now define a number $b$ and an ordinal $\gamma$ by $b=c_{p}\left(\beta_{i}\right) / c\left(\alpha_{i}\right)$ and $\gamma=\sum\left\{\omega^{e_{k}\left(\beta_{i}\right)} c_{k}\left(\beta_{i}\right) ; p<k \leqslant q\right\}$. By assumption, (S1) satisfies (C1); thus by Result $2 b$ is well defined and is positive. Furthermore, (II) tells us that $b$ and $\gamma$ are independent of the particular choice of $i$.

Now for any such $i$ we have $\sigma \alpha_{i}=\tau_{i}$, whence from the definition of $\tau_{i}$ we see that $\delta=e\left(r\left(\tau_{i}\right)\right)=e_{p}\left(\beta_{i}\right), p$ as above. Hence $\gamma<\sigma$, and we now put $\psi=\sigma b+\gamma$. We claim that $x=\psi$ is a solution of $(\mathrm{S} 1)$. For if $r\left(\alpha_{j}\right)>1$, we have $\psi \alpha_{j}=\omega^{\delta} \alpha_{j}=\tau_{j}=\beta_{j}$, since $\omega^{\delta} \leqslant \psi<\omega^{\delta+1}$. On the other hand, if $r\left(\alpha_{j}\right)=1$, then $\psi \alpha_{j}=\omega^{\delta} I\left(\alpha_{j}\right)+\omega^{\delta} b c\left(\alpha_{j}\right)+\gamma=\beta_{j}$. This proves our theorem.

COROLlary. Assume that the system 


$$
x \alpha_{j}=\beta_{j} ; \quad j<m,
$$

with $\alpha_{j}, \beta_{j}$ positive ordinals, has a solution. Then:

(1) If $r\left(\alpha_{j}\right)>1$ for every $j<m$, then $x=\psi$ is a solution of (S1) if and only if $\omega^{\delta} \leqslant \psi<\omega^{\delta+1}$ for some $\delta$ such that $\delta+e\left(\alpha_{j}\right)=e\left(\beta_{j}\right)$ for every $j<m$.

(2) If $r\left(\alpha_{i}\right)=1$ for some $i<m$, then (S1) has the unique solution

$$
x=\omega^{e_{p}\left(\beta_{i}\right)}\left[c_{p}\left(\beta_{i}\right) / c\left(\alpha_{i}\right)\right]+\sum\left\{\omega^{e_{k}\left(\beta_{i}\right)} c_{k}\left(\beta_{i}\right) ; p<k \leqslant q\right\},
$$

where $p=l\left(\alpha_{i}\right)-1, q=l\left(\beta_{i}\right)-1$.

THEOREM 2. The system

$$
\sum_{i=0}^{n-1} x_{i} \alpha_{i j}=\beta_{j} ; \quad j<m,
$$

with $\alpha_{i j}, \beta_{j}$ positive ordinals, has a solution if and only if there are numbers $i_{0}<i_{1}<\cdots<i_{s}<n$ and ordinals $\rho_{i_{k} j}, k \leqslant s$, with $\beta_{j}=\sum_{k=0}^{s} \rho_{i_{k} j}$ for every $j<m$, such that the $s+1$ systems

$$
x_{i_{k}} \alpha_{i_{k} j}=\rho_{i_{k} j} ; \quad j<m, k \leqslant s,
$$

all possess solutions.

Proof. The sufficiency of the condition is clear, since if each $\left(\mathrm{S}_{k}\right)$ has a solution $x_{i_{k}}=\psi_{k}$, then a solution of (S) is given by $x_{i}=\psi_{k}$ if $i=i_{k}$ for some $k \leqslant s$ and $x_{i}=0$ otherwise.

The necessity is proved by induction on $n$, the case $n=1$ being trivial. Thus take $t>1$ and assume that the necessity has been proved for every $n<t$. Suppose that the system (S) with $n=t$ has a solution $x_{i}=\gamma_{i}, i<t$. As each $\beta_{j}$ is positive, we must have $\gamma_{i}>0$ for some $i<t$. Let $i^{\circ}$ be the largest such $i$, and for each $j<m$ define $\rho_{j}, \tau_{j}$ by $\rho_{j}=\gamma_{i} \circ \alpha_{i \circ j}$ and $\tau_{j}=\sum_{i=0}^{i^{\circ}-1} \gamma_{i} \alpha_{i j}$. Then $\beta_{j}=\tau_{j}+\rho_{j}$, and the two systems

$$
\begin{aligned}
& \sum_{i=0}^{i^{\circ}-1} x_{i} \alpha_{i j}=\tau_{j} ; \quad j<m, \\
& x_{i} \circ \alpha_{i \circ j}=\rho_{j} ; \quad j<m,
\end{aligned}
$$

possess solutions.

As $i^{\circ}<t$ we can apply the induction hypothesis to $\left(\mathrm{S}^{*}\right)$, and the desired result is immediate.

Let us call a sequence of systems

$$
x_{i_{k}} \alpha_{i_{k} j}=\rho_{i_{k} j} ; \quad j<m, k \leqslant s
$$

"compatible" with a system

$$
\sum_{i=0}^{n-1} x_{i} \alpha_{i j}=\beta_{j} ; \quad j<m,
$$

if $i_{0}<i_{1}<\cdots<i_{s}<n$ and $\beta_{j}=\sum_{k=0}^{s} \rho_{i_{k} j}$ for every $j<m$, with each $\rho_{i_{k} j}$ positive.

Clearly every solution of (S) determines a compatible sequence, but it is also 
clear that a compatible sequence does not necessarily determine a unique solution of (S).

The proof of the following result is straightforward.

THEOREM 3. Assume that the system (S) (as above) has a solution. Then $x_{i}=\gamma_{i}$ is a solution of (S) if and only if some compatible sequence has a solution $x_{i_{k}}=\psi_{k}, k \leqslant s$, such that

(1) $\gamma_{i_{k}}=\psi_{k}$ for every $k \leqslant s$;

(2) $\gamma_{i}=0$ for every $i$ with $i_{s}<i<n$;

(3) $\gamma_{i} \alpha_{i j} \omega \leqslant \rho_{i_{k+1} j}$ for every $j<m$ and every $i$ with $i_{k}<i<i_{k+1}$ for some $k<s$.

The author wishes to thank the referee for his comments.

\section{REFERENCES}

1. S. Sherman, Some new properties of transfinite ordinals, Bull. Amer. Math. Soc. 47 (1941), 111-116. MR 2, 255.

2. W. Sierpiński, Cardinal and ordinal numbers, 2 nd rev. ed., Monografie Mat., vol. 34, PWN, Warsaw, 1965. MR 33 \#2549.

Department qf Mathematics, Institute of Advanced Studies, Australian National University, Canberra, Australia 